\title{
Spontaneous Breathing Trials With T-Piece or Pressure Support Ventilation
}

\author{
José Augusto S Pellegrini MD, Rafael B Moraes PhD, Juçara G Maccari MD, \\ Roselaine P de Oliveira PhD, Augusto Savi PhD, Rodrigo A Ribeiro PhD, Karen EA Burns PhD, \\ and Cassiano Teixeira $\mathrm{PhD}$
}

\author{
Introduction \\ Objectives \\ Methods \\ Data Sources and Searches \\ Study Selection \\ Outcome Measures \\ Data Extraction and Quality Assessment \\ Data Synthesis and Analysis \\ Results \\ Study Selection \\ Study Description \\ Outcomes Assessed \\ Study Protocols \\ Evidence Synthesis \\ Subgroup Analyses \\ Discussion \\ Summary of Evidence \\ Strengths and Limitations \\ Conclusions
}

Spontaneous breathing trials (SBTs) are among the most commonly employed techniques to facilitate weaning from mechanical ventilation. The preferred SBT technique, however, is still unclear. To clarify the preferable SBT (T-piece or pressure support ventilation [PSV]), we conducted this systematic review. We then searched the MEDLINE, EMBASE, SciELO, Google Scholar, CINAHL, ClinicalTrials.gov, and Cochrane CENTRAL databases through June 2015, without language restrictions. We included randomized controlled trials involving adult subjects being weaned from mechanical ventilation comparing T-piece with PSV and reporting (1) weaning failure, (2) re-intubation rate, (3) ICU mortality, or (4) weaning duration. Anticipating clinical heterogeneity among the included studies, we compared prespecified subgroups: (1) simple, difficult, or prolonged weaning and (2) subjects with COPD. We summarized the quality of evidence for intervention effects using the GRADE (Grading of Recommendations Assessment, Development, and Evaluation) methodology. We identified 3,674 potentially relevant studies and reviewed 23 papers in full. Twelve studies $(2,161$ subjects) met our inclusion criteria. Overall, the evidence was of very low to low quality. SBT technique did not influence weaning success (risk ratio 1.23 [0.94-1.61]), ICU mortality (risk ratio 1.11 [0.80-1.54]), or re-intubation rate (risk ratio 1.21 [0.90-1.63]). Prespecified subgroup analysis suggested that PSV might be superior to T-piece with regard to weaning success for simple-to-wean subjects (risk ratio 1.44 [1.11-1.86]). For the prolonged-weaning sub- 
group, however, T-piece was associated with a shorter weaning duration (weighted mean difference $\mathbf{- 3 . 0 8}[-5.24$ to -0.92$] \mathrm{d}$ ). In conclusion, low-quality evidence is available concerning this topic. PSV may be associated with lower weaning failure rates in the simple-to-wean subgroup. In contrast, in prolonged-weaning subjects, T-piece may be related to a shorter weaning duration, although this is at high risk of bias. Further study of the difficult-to-wean and COPD subgroups is required. Key words: weaning; mechanical ventilation; critical care. [Respir Care 2016;61(12):1693-1703. (C) 2016 Daedalus Enterprises]

\section{Introduction}

Successful weaning of patients from mechanical ventilation constitutes one of the most challenging tasks for ICU practitioners. Timely identification of patients who are capable of spontaneous breathing can shorten the mechanical ventilation duration and potentially reduce mechanical ventilation-related complications. ${ }^{1-5}$

Once a patient is deemed ready to breathe spontaneously, a screening test, called a spontaneous breathing trial (SBT), is usually performed, although the literature remains conflicted on this subject. ${ }^{6-10}$ An SBT is typically performed by disconnecting the patient from the ventilator and attaching a T-piece to the endotracheal tube. ${ }^{11}$ Some clinicians, however, prefer to use low levels of pressure support ventilation (PSV), or automatic tube compensation. ${ }^{8}$

Switching from continuous mandatory ventilation to spontaneous breathing can decrease left-ventricular performance and unmask latent left-ventricular heart failure. Concerns exist regarding the potential for SBT failure rates to be higher with T-piece SBTs than with low levels of PSV, possibly because of the increased expenditure of respiratory muscle energy ${ }^{12}$ and cardiogenic pulmonary edema secondary to the Müller maneuver. ${ }^{13}$ Although PSV

Drs Pellegrini, Maccari, de Oliveira, Savi, and Teixeira are affiliated with the Division of Critical Care, Hospital Moinhos de Vento (HMV), Porto Alegre, RS, Brazil. Drs Pellegrini and Moraes are affiliated with the Division of Critical Care, Hospital de Clínicas de Porto Alegre (HCPA), Porto Alegre, RS, Brazil. Drs de Oliveira and Teixeira are affiliated with the Medical School, Universidade Federal de Ciências da Saúde de Porto Alegre (UFCSPA), Porto Alegre, RS, Brazil. Dr Ribeiro is affiliated with the Institute for Education and Research, HMV, Graduate Program in Epidemiology, Universidade Federal do Rio Grande do Sul (UFRGS), Porto Alegre, RS, Brazil. Dr Burns is affiliated with the Interdepartmental Division of Critical Care Medicine, St. Michael's Hospital and the University of Toronto and the Li Ka Shing Knowledge Institute, Toronto, Canada.

The authors have disclosed no conflicts of interest.

Correspondence: José Augusto Santos Pellegrini MD, Rua Ramiro Barcelos, 910, Bloco D, 90035-001 - Porto Alegre, RS, Brazil. E-mail: joseaugusto.pellegrini@gmail.com.

DOI: $10.4187 /$ respcare. 04816 may be a less demanding SBT with regard to respiratory muscle effort and hydrostatic homeostasis, especially with the addition of PEEP to prevent the development of leftventricular heart failure, it may also dull the clinical picture of intolerance compared with that of unassisted $\mathrm{T}$ piece SBTs. ${ }^{13}$

Many trials have previously assessed this question, although heterogeneous methodological aspects and conflicting results limit adequate evidence appraisal. Previous meta-analyses have been conducted in this field but have not directly compared SBTs or used updated information. ${ }^{14}$

\section{Objectives}

Our objective was to clarify the preferred SBT technique (T-piece or low levels of PSV) for critically ill patients weaning from mechanical ventilation according to ICU mortality, re-intubation rates, weaning failure, and weaning duration.

\section{Methods}

We conducted a systematic review based on standard methods and reported our findings in accordance with the Preferred Reporting Items for Systematic Reviews and Meta-Analyses statement. ${ }^{15}$

\section{Data Sources and Searches}

We aimed to identify all randomized controlled trials (RCTs) assessing the efficacy and outcomes of T-piece compared with PSV trials in adult patients weaning from invasive mechanical ventilation.

We conducted electronic searches of the MEDLINE, EMBASE, Cochrane CENTRAL, CINAHL, SciELO, Google Scholar, and ClinicalTrials.gov databases for studies actively recruiting patients. All databases were searched from their inception to June 2015. Our MEDLINE search included the following terms and key words: ("weaning" OR "Ventilator Weaning"[MeSH]) AND ("Mechanical Ventilation" OR "Respiration, Artificial" [MeSH]) AND ("spontaneous breathing trial" OR "T-piece" OR "t tube"), using the Robinson and Dickersin RCT filter for PubMed. ${ }^{16}$ The electronic search strategy applied standard filters for the 


\section{SBTs WITH T-PIECE OR PSV}

identification of RCTs from each database. We screened the reference lists of retrieved publications for potentially eligible trials. We did not apply language restrictions.

\section{Study Selection}

We restricted our analysis to RCTs aiming to limit potential sources of bias. We excluded crossover trials and quasi-randomized trials. Regardless of specific weaning protocols, included trials had to compare between T-piece and PSV for conducting SBTs. We considered T-piece SBT to be the procedure of temporarily disconnecting a patient from the ventilator while maintaining an external oxygen supply, commonly by using a T-piece connected to the endotracheal tube. PSV was considered to be an SBT when employed in a systematic fashion, following a predefined protocol specifically designed to identify patients for extubation or, in the case of tracheostomized patients, for definitive removal from mechanical ventilation.

\section{Outcome Measures}

The outcomes assessed included (1) ICU mortality, (2) rate of re-intubation within $48 \mathrm{~h}$ following extubation, (3) weaning failure precluding extubation, and (4) weaning duration. We used authors' definitions for the post-randomization weaning duration.

\section{Data Extraction and Quality Assessment}

Two independent reviewers (JP and RM) screened the titles and abstracts of retrieved citations and the full texts of potentially eligible studies to identify trials that met our inclusion criteria. Data from each potentially relevant trial were independently extracted by the reviewers using a predefined data extraction form.

According to the Cochrane risk of bias tool, we appraised the adequacy of random sequence generation, the reporting of allocation concealment, the blinding of participants and outcome assessments, and the descriptions of losses to follow-up and exclusions; we still assessed adherence to the intention-to-treat principle. We solved disagreements by consulting a third reviewer (CT) when needed.

\section{Data Synthesis and Analysis}

Qualitative Analysis. We used a narrative summary approach to qualitatively describe the study characteristics and variations in quality indicators and to consider how these factors affected our understanding of the outcomes of the included RCTs.
Quantitative Analysis. We used the Cochrane Collaboration guidelines to conduct our meta-analysis. ${ }^{17}$ All statistical analyses were performed with Review Manager version 5.3 (Nordic Cochrane Center, Copenhagen, Denmark), the Cochrane Collaboration's software for preparing and updating Cochrane systematic reviews. We expressed the pooled effects estimates for binary and continuous variables using risk ratios and weighted mean differences with $95 \%$ CIs.

We tested for heterogeneity between studies using the Cochran $\mathrm{Q}$ and $\mathrm{I}^{2}$ tests. We predefined statistical heterogeneity as being low, intermediate, or high, correlated to $\mathrm{I}^{2}$ statistics $<25 \%$, from 25 to $50 \%$, or $>50 \%$, respectively. ${ }^{17}$ Meta-analyses with random-effects models were employed for all outcomes, due to anticipated clinical heterogeneity in terms of patient populations. We attempted to identify clinical factors as potential sources of heterogeneity, assessing for prespecified subgroups, including (1) weaning difficulty (simple, difficult, or prolonged weaning) and (2) COPD (vs subjects without COPD).

To assess the potential publication bias from small study effects, we constructed funnel plots displaying the log risk ratio on the horizontal axis and the SE of the log risk ratio on the vertical axis. We employed Egger's test to evaluate the risk for publication bias. We summarized the quality of evidence according to the Grading of Recommendations Assessment, Development, and Evaluation (GRADE) guidelines ${ }^{18}$ and reported them using the GRADE pro webbased tool.

\section{Results}

\section{Study Selection}

Our initial electronic search identified 3,674 abstracts. Of these, we excluded 3,651 because they did not describe RCTs, did not evaluate weaning techniques, were duplicate references, or were not relevant. We retrieved 23 studies for a more detailed, full-text analysis, and we excluded 11 of these studies ${ }^{19-29}$ (Fig. 1). We then identified 12 suitable studies comprising 2,161 subjects. Both reviewers completely agreed on the final selection of included studies. We also identified 2 ongoing RCTs, including one from our group, from the ClinicalTrials. gov database (Pellegrini, http://clinicaltrials.gov/show/ NCT01464567; Agarwal, http://clinicaltrials.gov/show/ NCT00911378 Accessed August 15, 2016).

\section{Study Description}

The included studies were published between 1994 and 2015 and were from 10 countries. Eight of the 12 included studies were single-center studies. ${ }^{30-37}$ One study was pub- 


\section{SBTs WITH T-PIECE OR PSV}

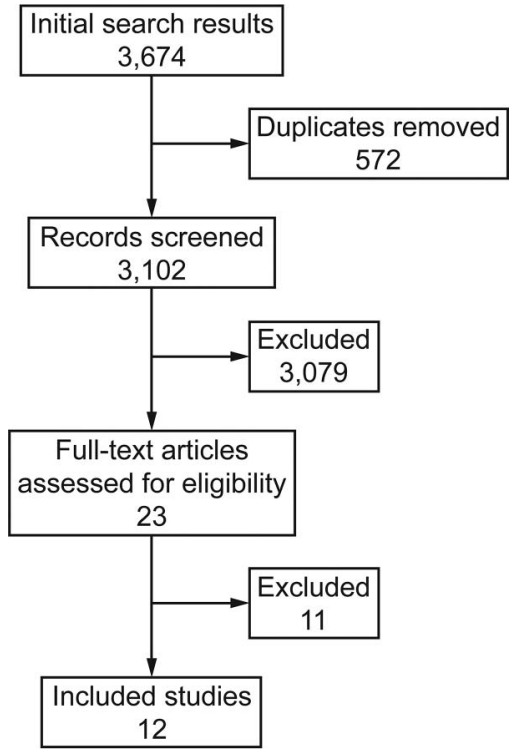

Fig. 1. Flow chart.

lished only in abstract form, ${ }^{37}$ and full details of the study were not available to the authors of this review.

Table 1 summarizes the components of the risk of bias assessment. Due to the nature of the intervention being studied, all studies were unblinded with regard to the patients enrolled and the outcomes assessed. Only 3 studies specified adequate random sequence generation, and 7 studies did not report adherence to the intention-to-treat principle. Our qualitative analysis of key study characteristics is summarized in Table 2.

\section{Outcomes Assessed}

All but 2 trials ${ }^{31}$ included in the study reported weaning failure outcome, which was defined as failure to extubate the subject immediately following the SBT. All but one ${ }^{36}$ study, apart from those assessing only tracheostomized subjects, reported 48-h re-intubation rates. Eight studies reported ICU mortality. ${ }^{32-35,37-39,41}$

\section{Study Protocols}

Brochard et $\mathrm{al}^{38}$ compared 3 different strategies for gradual weaning from mechanical ventilation. Beyond T-piece and PSV, the authors randomly assigned subjects to a third group using gradual titration of synchronized intermittent mandatory ventilation. T-piece SBTs were performed up to 8 times/d, progressing from 5 to $120 \mathrm{~min}$ in progressive steps. In subjects assigned to the PSV group, the PSV level was systematically adjusted 2 times/d to maintain a breathing frequency between 20 and 30 breaths/min in decremental steps of 2-4 $\mathrm{cm} \mathrm{H}_{2} \mathrm{O}$. Esteban et $\mathrm{al}^{40}$ compared once-a-day T-piece SBTs with
3 other methods, including intermittent mandatory ventilation, intermittent trials of spontaneous breathing (conducted two of more times a day if possible), in addition to PSV.

The study by Vitacca et $a^{41}$ differed from the other included studies by enrolling only difficult-to-wean tracheostomized subjects with COPD who required mechanical ventilation for at least $15 \mathrm{~d}$. This trial was conducted in 3 long-term weaning units, with subjects transferred from 24 ICUs after a range of 15-39 d on mechanical ventilation. The authors also compared their results with historical controls or an uncontrolled clinical practice. Jubran et al $^{34}$ assessed a similar population. Subjects who required mechanical ventilation for $>21 \mathrm{~d}$ in a long-term weaning unit were randomly assigned to unassisted breathing through a tracheostomy collar or to progressive reductions in PSV based on their breathing frequencies.

Six studies included simple-to-wean subjects, according to previously published definitions. . $30-32,35,36,39$ Three studies assessed difficult-to-wean subjects, ${ }^{33,38,40}$ and 2 studies included prolonged-weaning and tracheostomized subjects. ${ }^{34,41}$ Two additional studies evaluated postoperative subjects. ${ }^{35,37}$

In the studies that predefined the SBT duration, a 120min trial was most commonly employed (7 studies). Three authors reported progressively increasing duration of SBTs based on subjects' tolerance. ${ }^{34,38,41}$

Although included in various trials, subjects with COPD represented only a small fraction of the study population. Only 2 of the included studies specifically assessed subjects with $\mathrm{COPD}^{33,41}$; one of these also specifically enrolled prolonged-weaning subjects. ${ }^{41}$

\section{Evidence Synthesis}

T-piece SBTs were associated with a risk ratio $(95 \% \mathrm{CI})$ of $1.11(0.80-1.54)$ for ICU mortality and $1.21(0.90-$ 1.63) for the 48-h re-intubation rate (Figs. 2 and 3). The evidence from trials addressing these outcomes was considered very low to low quality based on the GRADE approach (Table 3). Study limitations, inconsistency, and imprecision contributed to downgrading the overall quality of evidence in the pooled RCTs.

For weaning failure, we found a risk ratio of 1.23 (0.94-1.61) (Fig. 4) with moderate to high heterogeneity $\left(\mathrm{I}^{2}=48 \%\right)$. When evaluating potential sources of clinical heterogeneity, we excluded prolonged-weaning studies from our analysis (8 studies remaining; 1,237 subjects) and noted a risk ratio of $1.47(1.17-1.84)$ favoring PSV with regard to weaning success. The $\mathrm{I}^{2}$ statistic for this analysis was $0 \%$, suggesting that prolonged-weaning studies represent an important source of clinical heterogeneity. 
Table 1. Risk of Bias Assessment

\begin{tabular}{|c|c|c|c|c|c|c|}
\hline Study & $\begin{array}{l}\text { Adequate } \\
\text { Sequence } \\
\text { Generation }\end{array}$ & $\begin{array}{c}\text { Allocation } \\
\text { Concealment }\end{array}$ & $\begin{array}{l}\text { Blinding of } \\
\text { Participants and } \\
\text { Investigators }\end{array}$ & $\begin{array}{l}\text { Blinding of } \\
\text { Outcome } \\
\text { Assessment }\end{array}$ & $\begin{array}{l}\text { Description of Losses } \\
\text { and Exclusions }\end{array}$ & $\begin{array}{l}\text { Intention to } \\
\text { Treat Analysis }\end{array}$ \\
\hline Brochard et al ${ }^{38}$ & Not clear & SNOSE & No & No & Yes & Yes \\
\hline Esteban et $\mathrm{al}^{39}$ & Yes & SNOSE & No & No & Yes & Not clear \\
\hline Esteban et $\mathrm{al}^{40}$ & Yes & SNOSE & No & No & Yes & Not clear \\
\hline Vitacca et $\mathrm{al}^{41}$ & Not clear & Not clear & No & No & Yes & Not clear \\
\hline Haberthür et al ${ }^{30}$ & Not clear & SNOSE & No & No & Yes & Yes \\
\hline Koksal et $\mathrm{al}^{31}$ & Not clear & Not clear & No & No & Yes & Yes \\
\hline Matić et al ${ }^{32}$ & Not clear & SNOSE & No & No & Yes & Yes \\
\hline Matić et al ${ }^{33}$ & Not clear & SNOSE & No & No & Yes & Not clear \\
\hline Jubran et $\mathrm{al}^{34}$ & Not clear & SNOSE & No & No & Yes & Yes \\
\hline Lourenço et $\mathrm{al}^{35}$ & Yes & No & No & No & Yes & Not clear \\
\hline Zhang et $\mathrm{al}^{36}$ & Not clear & Not clear & No & No & No & Not clear \\
\hline Chittawatanarat et al ${ }^{37}$ & Not clear & Not clear & No & No & Not clear & Not clear \\
\hline
\end{tabular}

SNOSE $=$ sequentially numbered, opaque, sealed envelopes

\section{Subgroup Analyses}

Weaning Difficulty. Seven studies (1,600 subjects) focused on simple weaning, which was defined as success on the first SBT in the absence of previous weaning failure. PSV in this population was associated with better outcomes related to weaning success (risk ratio $=1.44,1.11-$ $1.86 ; \mathrm{I}^{2}=0 \%$ ) but not with lower re-intubation or ICU mortality rates. Three studies (197 subjects) specifically assessed difficult-to-wean subjects. In this subgroup, significant differences in clinical outcomes were not found between the alternative SBT techniques.

Only 2 studies, comprising 364 subjects, focused on prolonged-weaning subjects. Whereas the SBT technique had no effect on weaning failure rate or ICU mortality, T-piece was associated with a shorter weaning duration (weighted mean difference $=-3.08(-5.24$ to -0.92$) \mathrm{d}$ of weaning) than that associated with PSV (Fig. 5). These aforementioned outcomes had an $\mathrm{I}^{2}=0 \%$ in this subgroup.

COPD Subjects. In the 12 included studies, we identified 338 subjects with COPD, although 3 studies did not specifically report this condition.

Between the 2 studies enrolling exclusively subjects with COPD, we identified remarkable clinical heterogeneity preventing pooling. Whereas the study of Matic et al ${ }^{33}$ included difficult-to-wean subjects with COPD (defined as one failed weaning attempt), the Vitacca study ${ }^{41}$ included tracheostomized subjects ventilated for $\geq 15 \mathrm{~d}$. The risk ratios in the Matić and Vitacca studies for weaning failure with T-piece SBTs were $1.61(0.82-3.16)$ and 0.86 (0.33-2.21), respectively, and risk ratios for ICU mortality were $2.06(0.41-10.47)$ and $0.67(0.12-3.67)$, respectively.
We performed funnel-plot analysis for each outcome and did not identify publication bias. The funnel plot for weaning failure is shown in Figure 6. Egger's test did not suggest publication bias $(P=.37)$.

\section{Discussion}

\section{Summary of Evidence}

T-piece and PSV are 2 of the most commonly used techniques when conducting SBTs in clinical practice today. Nevertheless, existing evidence directly comparing these 2 approaches is sparse, heterogeneous, and of poor overall quality. Small study populations with low event rates, variability among the applied SBT techniques, and remarkably different populations limit the pooling and adequate interpretation of evidence.

T-piece and PSV techniques have theoretical singularities that may influence bedside judgment when choosing one SBT technique over another. When using T-piece SBTs, one might be looking for specificity and thus might proceed with extubation only for those patients able to tolerate the hemodynamic perturbations of this disturbing test. ${ }^{13,42}$ Also, previous studies have shown that the postextubation work of breathing could be more closely paralleled by unassisted breathing (as in a T-piece trial) than by a low-pressure support trial. ${ }^{43}$

By contrast, Ezingeard et $\mathrm{al}^{44}$ demonstrated that more subjects could be successfully extubated after a PSV SBT, including some subjects who previously failed a T-piece SBT. These findings are supported by moderate-quality evidence with regard to simple-to-wean subjects, for whom PSV might be associated with reduced weaning failure rates, not adversely influencing re-intubation rates. 


\section{SBTs With T-PIECE OR PSV}

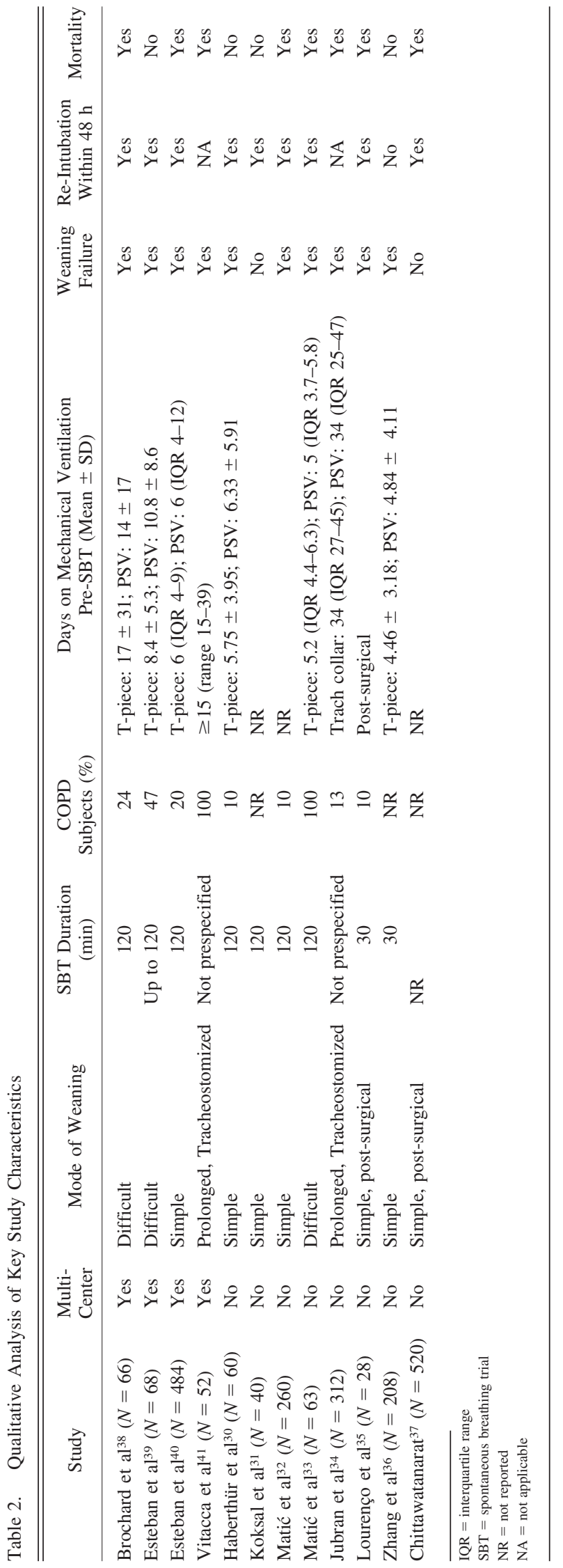

In contrast, low-quality evidence suggests that prolonged-weaning subjects ${ }^{31,37}$ appear to benefit from T-piece SBTs in terms of weaning duration. In these subjects, progressive steps toward predetermined reductions in PSV according to the subject's tolerance may prolong the duration of mechanical ventilation, potentially increasing the risk for mechanical ventilation-related complications. However, we observed that the few studies including subjects who experienced such events found that the SBT technique has no influence on mortality in this subgroup.

Patients with COPD represent a growing population worldwide, remaining as one of the most prevalent conditions leading to respiratory failure and consequent mechanical ventilation. ${ }^{45-47}$ These individuals represent some of the most challenging groups to wean from mechanical ventilation $^{48}$; paradoxically, this population is underrepresented in RCTs. Two groups of authors evaluated subjects with COPD exclusively, but their studies included markedly different profiles. One group of authors ${ }^{33}$ enrolled difficult-to-wean subjects with COPD, and the other ${ }^{41}$ focused on tracheostomized, prolonged-weaning subjects in long-term weaning units. Recognizing that clinical heterogeneity would hinder the interpretation of findings, we decided not to pool these results.

A relevant aspect that should be kept in mind concerns the consideration of SBTs as an intervention rather than a diagnostic test trying to identify patients who are potential candidates for extubation, predicting tolerance of unassisted breathing. In the latter approach, questions focus on diagnostic accuracy, and then weaning failure or re-intubation rates are important end points for describing weaning trial sensitivity or specificity.

Nevertheless, the assessment of diagnostic properties of SBTs for predicting successful extubation is not straightforward. Extubation failure rates are widely reported as being around 15-20\%, which makes specificity of the trial for predicting successful extubation only $80-85 \%$. On the opposite side, test sensitivity (the proportion of patients able to tolerate extubation despite failing the weaning test) is difficult to evaluate because patients who fail a weaning test are usually not extubated. Furthermore, criteria for termination of a weaning trial and even definitions of test failure are essentially subjective and clinician-dependent, potentially biasing outcomes beyond the test itself. Accordingly, we decided to stay in line with previous studies and assess the clinical impact of SBTs as an intervention in important outcomes, beyond its diagnostic role in predicting patient tolerance to mechanical ventilation discontinuation.

Overall, our results are consistent with those of a Cochrane review ${ }^{14}$ and are applicable to the general weaning population encountered by clinicians in clinical practice. Our review, however, adds important additional information from 4 published RCTs, increasing the size of the included population (2,161 subjects here vs 1,208 subjects 


\section{SBTs WITH T-PIECE OR PSV}

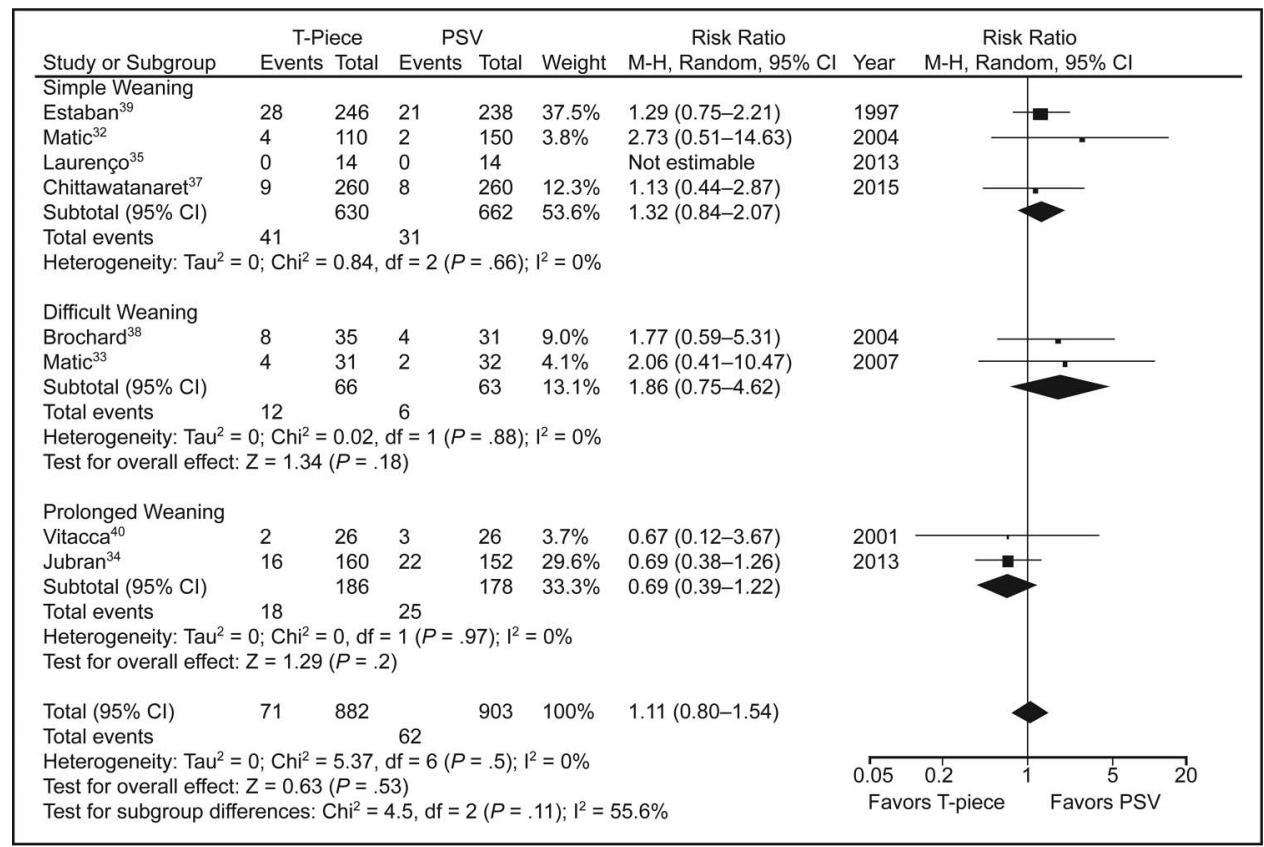

Fig. 2. ICU mortality. M-H = Maentel-Haentzel; PSV = pressure support ventilation.

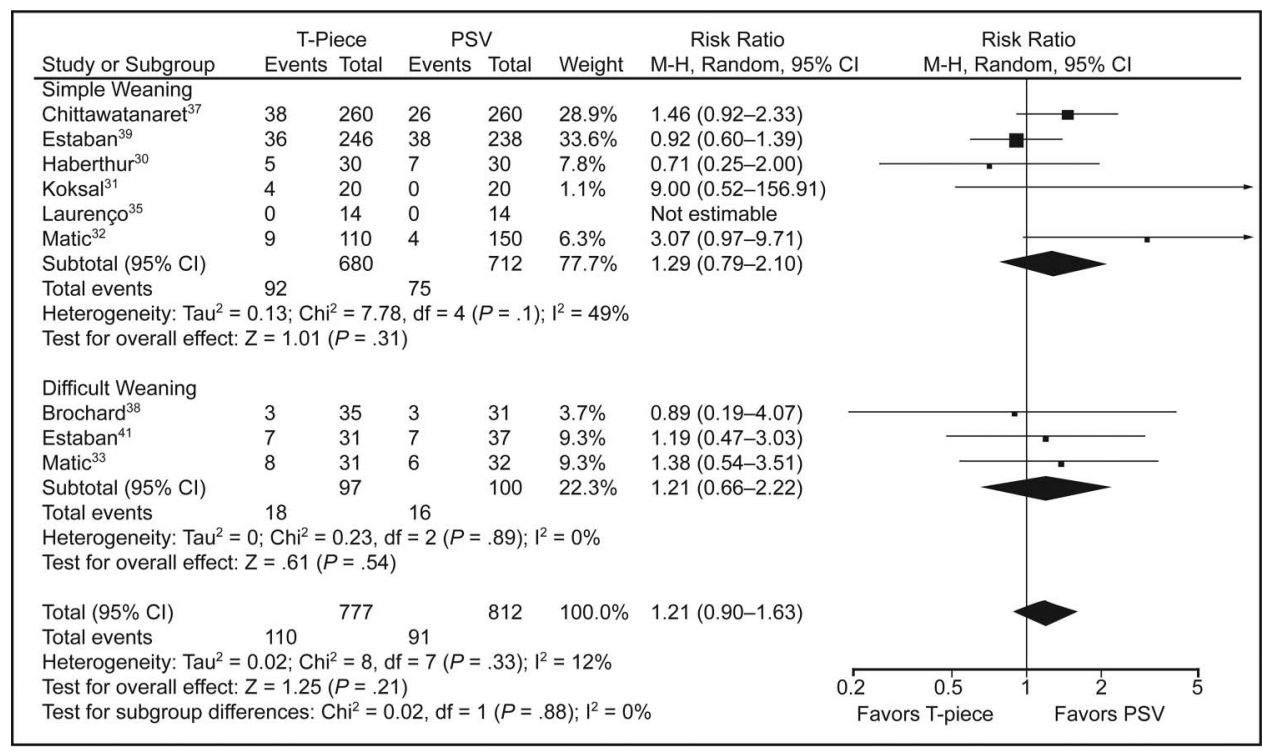

Fig. 3. 48-h reintubation rate. $\mathrm{M}-\mathrm{H}=$ Maentel-Haentzel; PSV = pressure support ventilation.

previously analyzed). In addition, we defined an a priori subgroup analysis aimed at identifying different effects of the alternative SBT techniques based on weaning difficulty and the presence of COPD. Very low to low overall quality of evidence strongly limits definitive findings in this field.

\section{Strengths and Limitations}

We conducted a systematic search of several databases without language restrictions to identify all RCTs compar- ing T-piece and PSV SBT techniques in weaning subjects. We employed standardized techniques to assess risk of bias and overall quality of evidence.

Our review has several limitations that reduce the strength of inferences that can be made. First, quality assessment permits classifying the evidence as very low to low quality. Although some aspects of bias assessment are not relevant in this area (eg, blinding of patients and investigators in necessarily unblinded trials), others, such as sequence generation and allocation concealment, reveal 


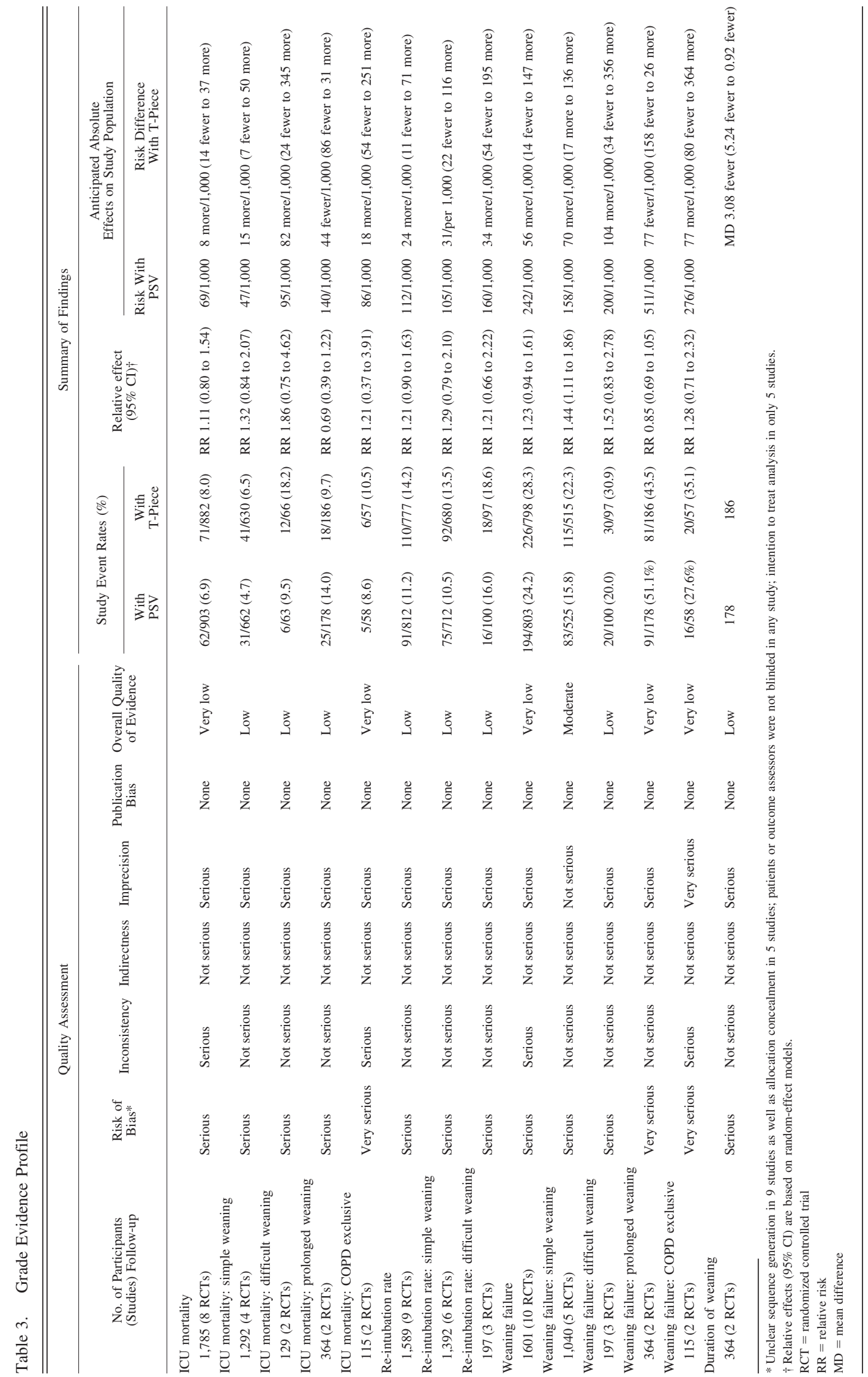




\section{SBTs WITH T-PIECE OR PSV}

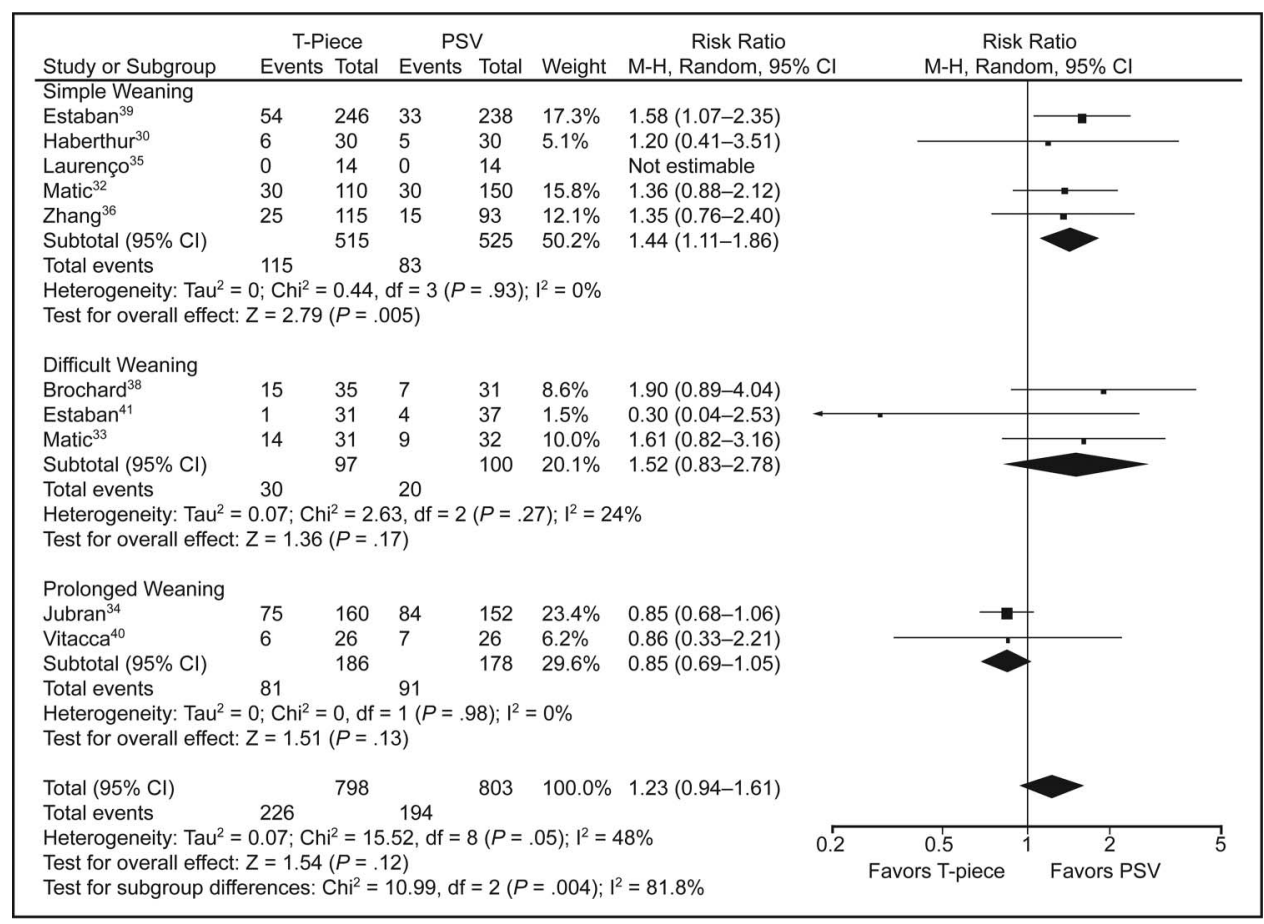

Fig. 4. Weaning failure. $\mathrm{M}-\mathrm{H}=$ Maentel-Haentzel; PSV = pressure support ventilation.

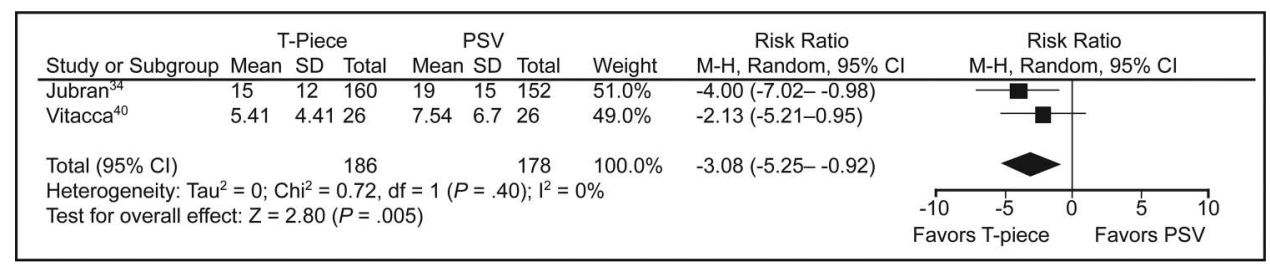

Fig. 5. Forest plot comparing T-piece and pressure support ventilation (PSV) for weaning duration. $\mathrm{M}-\mathrm{H}=\mathrm{Maentel-Haentzel}$.

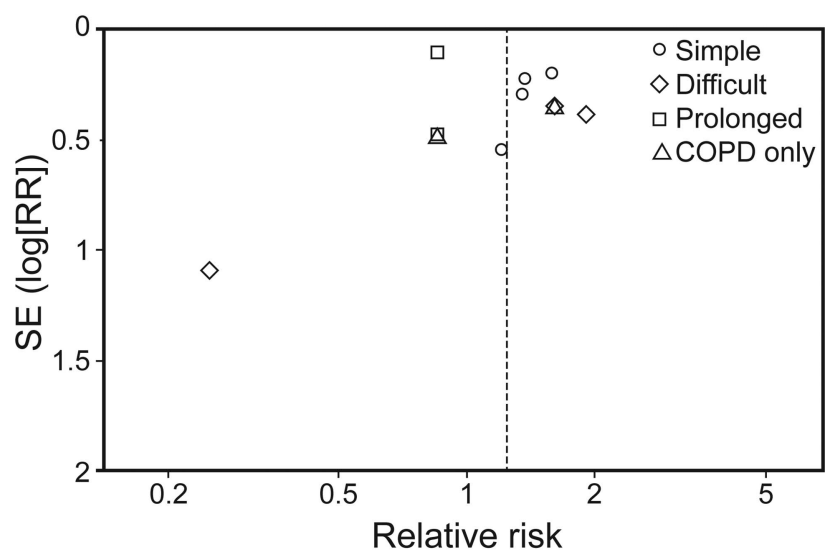

Fig. 6. Funnel plot for weaning failure outcome.

methodological issues that may impact study findings. Imprecision of available data was an important source of downgrading of evidence for many outcomes. Second, we identified important clinical heterogeneity among studies that hindered the pooling of estimates and limited the generalizability of our findings. Some aspects related to mechanical ventilation settings (different pressure support levels and protocols, adjunctive use of PEEP) certainly contribute to conflicting results. This could be considered one of the most important issues in this review. Third, subgroup analysis should be interpreted with caution, accordingly to study populations and outcomes reported. Finally, the difficult-to-wean subject and COPD subject subgroups remain scarcely studied, limiting conclusions in these areas.

\section{Conclusions}

The quality of available evidence precludes definitive conclusions about assessed outcomes. Low-quality evidence suggests that PSV SBTs may result in lower weaning failure rates in simple-to-wean subjects but do not affect re-intubation rates or other important outcomes. Conversely, in prolonged-weaning subjects, a T-piece may reduce the weaning duration compared with PSV SBTs. 


\section{SBTs WITH T-PIECE OR PSV}

Future trials should compare SBT techniques in difficultto-wean and COPD subjects.

\section{REFERENCES}

1. Klompas M, Anderson D, Trick W, Babcock H, Kerlin MP, Li L, et al. The preventability of ventilator-associated events: the CDC Prevention Epicenters Wake Up and Breathe Collaborative. Am J Respir Crit Care Med 2015;191(3):292-301.

2. Boles JM, Bion J, Connors A, Herridge M, Marsh B, Melot C, et al. Weaning from mechanical ventilation. Eur Respir J 2007;29(5):10331056.

3. Peñuelas Ó, Thille AW, Esteban A. Discontinuation of ventilatory support: new solutions to old dilemmas. Curr Opin Crit Care 2015; 21(1):74-81.

4. Balas MC, Vasilevskis EE, Olsen KM, Schmid KK, Shostrom V, Cohen MZ, et al. Effectiveness and safety of the awakening and breathing coordination, delirium monitoring/management, and early exercise/mobility bundle. Crit Care Med 2014;42(5):1024-1036.

5. Trogrlić Z, van der Jagt M, Bakker J, Balas MC, Ely EW, van der Voort PH, Ista E. A systematic review of implementation strategies for assessment, prevention, and management of ICU delirium and their effect on clinical outcomes. Crit Care 2015;19:157.

6. Tanios MA, Nevins ML, Hendra KP, Cardinal P, Allan JE, Naumova EN, Epstein SK. A randomized, controlled trial of the role of weaning predictors in clinical decision making. Crit Care Med 2006; 34(10):2530-2535.

7. Teixeira C, Zimermann Teixeira PJ, Hohër JA, de Leon PP, Brodt SF, da Siva Moreira J. Serial measurements of f/VT can predict extubation failure in patients with $\mathrm{f} / \mathrm{VT}<$ or $=105$ ? J Crit Care 2008;23(4):572-576.

8. Cohen JD, Shapiro M, Grozovski E, Singer P. Automatic tube compensation-assisted respiratory rate to tidal volume ratio improves the prediction of weaning outcome. Chest 2002;122(3):980-984.

9. Namen AM, Ely EW, Tatter SB, Case LD, Lucia MA, Smith A, et al. Predictors of successful extubation in neurosurgical patients. Am J Respir Crit Care Med 2001;163(3 Pt 1):658-664.

10. Krishnan JA, Moore D, Robeson C, Rand CS, Fessler HE. A prospective, controlled trial of a protocol-based strategy to discontinue mechanical ventilation. Am J Respir Crit Care Med 2004;169(6): 673-678.

11. Rubini F, Zanotti E, Nava S. Ventilatory techniques during weaning. Monaldi Arch Chest Dis 1994;49(6):527-529.

12. dos Santos LJ, Hoff FC, Condessa RL, Kaufmann ML, Vieira SR. Energy expenditure during weaning from mechanical ventilation: is there any difference between pressure support and T-tube? J Crit Care 2011;26(1):34-41.

13. Cabello B, Thille AW, Roche-Campo F, Brochard L, Gómez FJ, Mancebo J. Physiological comparison of three spontaneous breathing trials in difficult-to-wean patients. Intensive Care Med 2010; 36(7):1171-1179.

14. Ladeira MT, Vital FM, Andriolo RB, Andriolo BN, Atallah AN, Peccin MS. Pressure support versus T-tube for weaning from mechanical ventilation in adults. Cochrane Database Syst Rev 2014; (5):CD006056.

15. Moher D, Liberati A, Tetzlaff J, Altman DG. Preferred reporting items for systematic reviews and meta-analyses: the PRISMA statement. PLoS Med 2009;6(7):e1000097.

16. Robinson KA, Dickersin K. Development of a highly sensitive search strategy for the retrieval of reports of controlled trials using PubMed. Int J Epidemiol 2002;31(1):150-153.

17. Higgins JPT, Green S, editors. Cochrane handbook for systematic reviews of interventions, version 5.1.0. The Cochrane Collaboration. www.handbook.cochrane.org
18. Atkins D, Best D, Briss PA, Eccles M, Falck-Ytter Y, Flottorp S, et al. Grading quality of evidence and strength of recommendations. BMJ 2004;328(7454):1490.

19. Koh Y, Hong SB, Lim CM, Lee SD, Kim WS, Kim DS, Kim WD. Effect of an additional 1-hour T-piece trial on weaning outcome at minimal pressure support. J Crit Care 2000;15(2):41-45.

20. Mekontso-Dessap A, de Prost N, Girou E, Braconnier F, Lemaire F, Brun-Buisson C, Brochard L. B-type natriuretic peptide and weaning from mechanical ventilation. Intensive Care Med 2006;32(10):15291536.

21. Colombo T, Boldrini AF, Juliano SR, Juliano MC, Houly JG, Gebara $\mathrm{OC}$, et al. [Implementation, assessment and comparison of the T-tube and pressure-support weaning protocols applied to the intensive care unit patients who had received mechanical ventilation for more than 48 hours]. Rev Bras Ter Intensiva 2007;19(1):31-37.

22. Molina-Saldarriaga FJ, Fonseca-Ruiz NJ, Cuesta-Castro DP, Esteban A, Frutos-Vivar F. [Spontaneous breathing trial in chronic obstructive pulmonary disease: continuous positive airway pressure (CPAP) versus T-piece]. Med Intensiva 2010;34(7):453-458.

23. Gnanapandithan K, Agarwal R, Aggarwal AN, Gupta D. Weaning by gradual pressure support (PS) reduction without an initial spontaneous breathing trial (SBT) versus PS-supported SBT: a pilot study. Rev Port Pneumol 2011;17(6):244-252.

24. Bien MY, Shui Lin Y, Shih CH, Yang YL, Lin HW, Bai KJ, et al. Comparisons of predictive performance of breathing pattern variability measured during T-piece, automatic tube compensation, and pressure support ventilation for weaning intensive care unit patients from mechanical ventilation. Crit Care Med 2011;39(10):2253-2262.

25. Wang J, Ma Y, Fang Q. Extubation with or without spontaneous breathing trial. Crit Care Nurse 2013;33(6):50-55.

26. DiNino E, Gartman EJ, Sethi JM, McCool FD. Diaphragm ultrasound as a predictor of successful extubation from mechanical ventilation. Thorax 2014;69(5):423-427.

27. Ma YM, Liu YN, Pan L. [The effect of spontaneous breathing trial on weaning from ventilators]. Zhonghua Jie $\mathrm{He} \mathrm{He} \mathrm{Hu} \mathrm{Xi} \mathrm{Za} \mathrm{Zhi}$ 2010;33(3):179-182.

28. Kuhlen R, Max M, Dembinski R, Terbeck S, Jürgens E, Rossaint R. Breathing pattern and workload during automatic tube compensation, pressure support and T-piece trials in weaning patients. Eur J Anaesthesiol 2003;20(1):10-16.

29. Emmerich J, Vicêncio S, Siqueira H, Simão A, Bernhoeft C, Souza W. A comparison among three methods of weaning ventilatory support: T-piece versus S-IMV versus PSV. Rev Bras Ter Intensiva 1997;9(4):167-174.

30. Haberthür C, Mols G, Elsasser S, Bingisser R, Stocker R, Guttmann J. Extubation after breathing trials with automatic tube compensation, T-tube, or pressure support ventilation Acta Anaesthesiol Scand 2002;46(8):973-979.

31. Koksal GM, Sayilgan C, Sen O, Oz H. The effects of different weaning modes on the endocrine stress response. Crit Care 2004; 8(1):R31-R34.

32. Matić I, Majerić-Kogler V. Comparison of pressure support and T-tube weaning from mechanical ventilation: randomized prospective study. Croat Med J 2004;45(2):162-166.

33. Matić I, Danić D, Majerić-Kogler V, Jurjević M, Mirković I, Mrzljak Vucinić N. Chronic obstructive pulmonary disease and weaning of difficult-to-wean patients from mechanical ventilation: randomized prospective study. Croat Med J 2007;48(1):51-58.

34. Jubran A, Grant BJ, Duffner LA, Collins EG, Lanuza DM, Hoffman LA, Tobin MJ. Effect of pressure support vs unassisted breathing through a tracheostomy collar on weaning duration in patients requiring prolonged mechanical ventilation: a randomized trial. JAMA 2013;309(7):671-677. 


\section{SBTs WITH T-PIECE OR PSV}

35. Lourenço IS, Franco AM, Bassetto S, Rodrigues AJ. Pressure support-ventilation versus spontaneous breathing with "T-Tube" for interrupting the ventilation after cardiac operations. Rev Bras Cir Cardiovasc 2013;28(4):455-461.

36. Zhang B, Qin YZ. Comparison of pressure support ventilation and T-piece in determining rapid shallow breathing index in spontaneous breathing trials. Am J Med Sci 2014;348(4):300-305.

37. 35 th international symposium on intensive care and emergency medicine. Crit Care 2015;19(Suppl 1):P1-P578.

38. Brochard L, Rauss A, Benito S, Conti G, Mancebo J, Rekik N, et al. Comparison of three methods of gradual withdrawal from ventilatory support during weaning from mechanical ventilation. Am J Respir Crit Care Med 1994;150(4):896-903.

39. Esteban A, Alía I, Gordo F, Fernández R, Solsona JF, Vallverdú I, et al. Extubation outcome after spontaneous breathing trials with T-tube or pressure support ventilation: the Spanish Lung Failure Collaborative Group. Am J Respir Crit Care Med 1997;156(2 Pt 1):459-465.

40. Esteban A, Frutos F, Tobin MJ, Alía I, Solsona JF, Valverdú I, et al. A comparison of four methods of weaning patients from mechanical ventilation: Spanish Lung Failure Collaborative Group. N Engl J Med 1995;332(6):345-350.

41. Vitacca M, Vianello A, Colombo D, Clini E, Porta R, Bianchi L, et al. Comparison of two methods for weaning patients with chronic obstructive pulmonary disease requiring mechanical ventilation for more than 15 days. Am J Respir Crit Care Med 2001;164(2):225230.
42. Teixeira C, Teixeira PJ, de Leon PP, Oliveira ES. Work of breathing during successful spontaneous breathing trial. J Crit Care 2009;24(4): 508-514.

43. Straus C, Louis B, Isabey D, Lemaire F, Harf A, Brochard L. Contribution of the endotracheal tube and the upper airway to breathing workload. Am J Respir Crit Care Med 1998;157(1):23-30.

44. Ezingeard E, Diconne E, Guyomarc'h S, Venet C, Page D, Gery P, et al. Weaning from mechanical ventilation with pressure support in patients failing a T-tube trial of spontaneous breathing. Intensive Care Med 2006;32(1):165-169.

45. Choi SM, Lee J, Park YS, Lee CH, Lee SM, Yim JJ, et al. Prevalence and global initiative for chronic obstructive lung disease group distribution of chronic obstructive pulmonary disease detected by preoperative pulmonary function test. PLoS One 2015;10(1):e0115787.

46. Davis KJ, Landis SH, Oh YM, Mannino DM, Han MK, van der Molen T, et al. Continuing to Confront COPD International Physician Survey: physician knowledge and application of COPD management guidelines in 12 countries. Int J Chron Obstruct Pulmon Dis 2015;10:39-55.

47. Raluy-Callado M, Lambrelli D, MacLachlan S, Khalid JM. Epidemiology, severity, and treatment of chronic obstructive pulmonary disease in the United Kingdom by GOLD 2013. Int J Chron Obstruct Pulmon Dis 2015;10:925-937.

48. Teixeira C, Maccari JG, Vieira SR, Oliveira RP, Savi A, Machado AS, et al. Impact of a mechanical ventilation weaning protocol on the extubation failure rate in difficult-to-wean patients. J Bras Pneumol 2012;38(3):364-371 